\title{
MICRORAMAN STUDY OF Ge/Si QUANTUM RINGS AND DOTS
}

\author{
V.I. Mashanov ${ }^{\mathrm{a}}$, H.-H. Cheng ${ }^{\mathrm{a}, \mathrm{b}}$, L.-J. Chen ${ }^{\mathrm{c}}$, C.-T. Chia ${ }^{\mathrm{c}}$ \\ ${ }^{a}$ Center for Condensed Matter Sciences, National Taiwan University, Taipei106, Taiwan R.O.C. \\ ${ }^{b}$ Graduate Institute of Electronic Engineering, National Taiwan University, Taipei106, Taiwan \\ R.O.C. \\ ${ }^{\mathfrak{c}}$ Department of Physics, National Taiwan Normal University, Taipei 106, Taiwan R.O.C.
}

$\mathrm{Ge} / \mathrm{Si}$ nanostructure has attracted great attention in recent year for its potential application in optical-electronic devices. As growth techniques advanced, the structure evolves from two-dimensional heterojunction to zero-dimensional quantum dots. In a recent investigation, ring shape structure with a hole in the middle is observed [1,2]. Nevertheless, the physical properties are less investigated.

In this report we present the fabrication and optical measurement of $\mathrm{Ge} / \mathrm{Si}$ nanostructure. Atomic force microscopy (AFM) is employed to probe the surface morphology. It shows both quantum dots (QDs) and ring shape Ge nanostructure (hereafter we call it quantum ring QRs). MicroRaman is performed to characterize the strain and composition QD and QR.

The samples are grown by solid source molecular beam epitaxy (MBE) with electron beam evaporators for both $\mathrm{Ge}$ and $\mathrm{Si}$. N-type $\mathrm{Si}(100)$ wafer with resistivity of $1-10 \mathrm{om}-\mathrm{cm}$ is used. Prior to the growth, the wafer is flush with low $\mathrm{Si}$ flux to remove the thin $\mathrm{SiO}_{2}$ layer. The procedure repeats several times until the sharp 2 by 1 pattern is observed by the RHEED to ensure the clean surface. For all the samples, thin Si buffer layer of $30 \mathrm{~nm}$ is deposited at $700 \mathrm{C}$. Low growth flux is employed for the structure with a rate of $0.01 \mathrm{~nm} / \mathrm{s}$ and $0.06 \mathrm{~nm} / \mathrm{s}$ for $\mathrm{Ge}$ and $\mathrm{Si}$ respectively. The structure consists of (a) a layer of $\mathrm{Ge}$ of $0.7 \mathrm{~nm}$, (b) a thin layer of $0.05 \mathrm{~nm}$ of Ge, and (c) Si cap layer of $2.5 \mathrm{~nm}$. In between layer (a) and (b), both Si and $\mathrm{Ge}$ shutter is closed for five minute to ensure the uniformity of the Ge layer [1]. Four samples are grown at various temperatures of $6400^{\circ} \mathrm{C}, 660{ }^{\circ} \mathrm{C}, 680^{\circ} \mathrm{C}$ and $700{ }^{\circ} \mathrm{C}$.

A typical AFM picture is shown in Figure 1. In the image, both quantum dots (dotted circle) and rings (solid circle) are observed. For the dots, in the four samples, the diameters range from 200 to $300 \mathrm{~nm}$ with a height of $3-10 \mathrm{~nm}$. For the QRs, the diameter is larger than the dots ranging from $250 \mathrm{~nm}$ to $560 \mathrm{~nm}$ but with smaller height ranging from $1 \mathrm{~nm}$ to $16 \mathrm{~nm}$. The dots is formed through (a) strain relaxation process and (b) alloying to reduce the strain energy of the epilayer. In the former factor, the 2D layer evolves into 3D islands with either dome or pyramid shape depending on the thickness of the deposition. For the second factor, Ge intermixes with the underlying Si resulting $\mathrm{Si}_{1-\mathrm{x}} \mathrm{Ge}_{\mathrm{x}}$ alloy. Ge composition depends on the growth temperature, for the growth temperature employed in this studied, Ge range from $68 \%$ $\left(640{ }^{\circ} \mathrm{C}\right)$ to $50 \%\left(\mathrm{~T}=700{ }^{\circ} \mathrm{C}\right)[3,4]$. As dot becomes larger (or merge with the neighbouring dot), it transform into the ring shape to minimize the total energy.

MicroRaman measurements were performed at room temperature. Wavelength of 514.5 $\mathrm{nm}$ from Art laser was used as the excitation source. Focusing spot is about $1 \mu \mathrm{m}$ with a spectrum resolution of $0.5 \mathrm{~cm}^{-1}$. For the sample structure used in this studied, the optical spot cover both QDs and QRs. The spectra, after the background subtraction of the wafer, are shown in Fig. 2. Two clear features are observed at wavelength around $300 \mathrm{~cm}^{-1}$ and $413 \mathrm{~cm}^{-1}$, corresponding to the $\mathrm{Ge}-\mathrm{Ge}$, and $\mathrm{Ge}-\mathrm{Si}$ phonon mode. (SiSi phonon mode at $520 \mathrm{~cm}^{-1}$ does not plot in the figure as due to the strong intensity) From the plot, it shows two characteristics. Firstly, the intensity of both $\mathrm{Ge}-\mathrm{Ge}$ and $\mathrm{Si}-\mathrm{Ge}$ modes decrease as growth temperature increase. Secondly, the peak position of the Si-Ge line remains at the same position within $\pm 1 \mathrm{~cm}^{-1}$. To analyze the change of Ge composition, we take the intensity ratio of $\mathrm{Ge}-\mathrm{Ge}$ mode and $\mathrm{Si}-\mathrm{Ge}$ mode. The result is depicted in Fig. 3. It shows that the ratio decreases with increasing growth 
temperature. As $\mathrm{Ge}$ is deposited on $\mathrm{Si}$, this reflects a larger degree of intermixing at higher growth temperature, consistent with the previous report on QDs [3]. (In the study, Ge composition in the dots is $68 \%$ grown at $640{ }^{\circ} \mathrm{C}$ and $50 \%$ at $700{ }^{\circ} \mathrm{C}$ ) The change of the composition should give a shift in the peak energy. However, a clear shift is not resolved. This may due to the different mixing mechanism in the QRs.

Acknowledgements

This work is funded by National Science Council (Taiwan, ROC).

References

1 J. Cui, Q. He, X. M. Jiang, Y. L. Fan, X. J. Yang, F. Xue, Z. M. Jiang, Appl. Phys. Lett. 83, 2907 (2003).

2. S. W. Lee, L. J. Chen, P. S. Chen, M.-J. Tsai, C. W. Liu, T. Y. Chien, C. T. Chia, Appl. Phys. Lett. 83, $5283(2003)$.

3 G. Capellini, M. De Seta, F. Evangelisti, Appl. Phys. Lett. 78,303 (2001).

4. X. Z. Liao, J. Zou, D. J. H. Cockayne, J. Qin, Z. M. Jiang, X. Wang, R. Leon Phys. Rew. B 60, 15605 (1999).

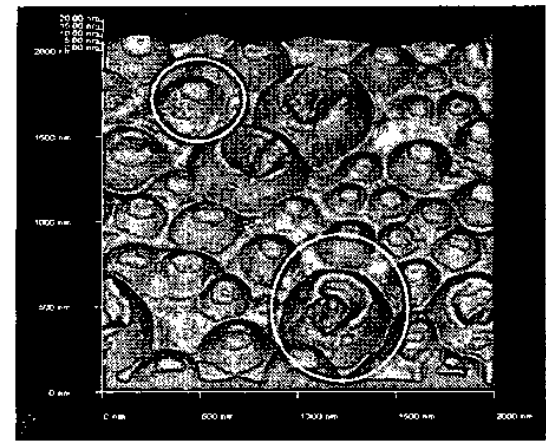

Fig. 1 AFM image showing both QR (solid circle) and QD (dotted circle).

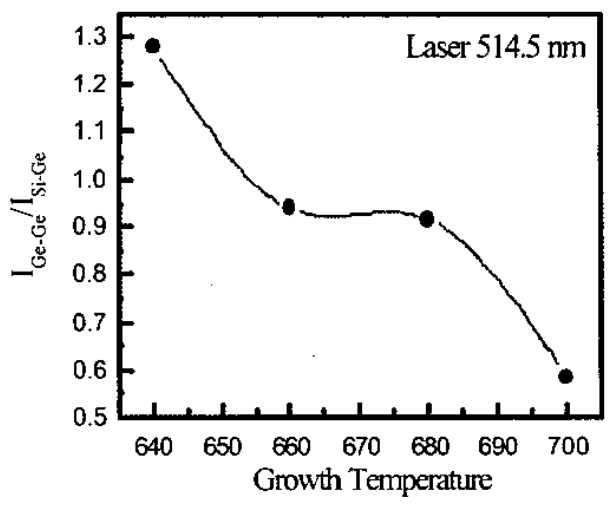

Fig. 3 Intensity ratio of $\mathrm{GeGe}$ mode to SiGe mode

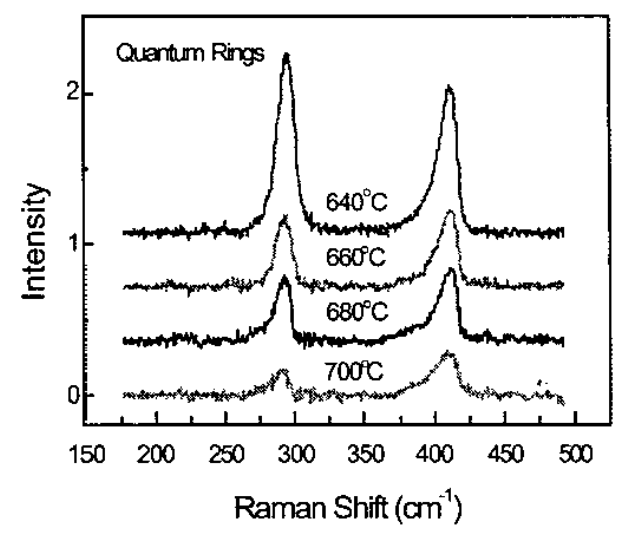

Fig. 2: (a) The Raman signals of the four samples with the growth temperatures $640^{\circ} \mathrm{C}, 660^{\circ} \mathrm{C}, 680^{\circ} \mathrm{C}$ and $700{ }^{\circ} \mathrm{C}$. 